\title{
Weakly Coupled Antiferromagnetic Quantum Spin Chains
}

\author{
Ziqiang Wang \\ Department of Physics, Boston College, Chestnut Hill, MA 02167
}

\begin{abstract}
Quasi-one-dimensional quantum antiferromagnets formed by a $d$-dimensional hypercubic lattice of weakly coupled spin-1/2 antiferromagnetic Heisenberg chains are studied by combining exact results in one-dimension and renormalization group analyses of the interchain correlations. It is shown that $d$-dimensional magnetic long-range order develops at zero-temperature for infinitesimal antiferromagnetic or ferromagnetic interchain couplings. In the presence of weak bond alternations, the order-disorder transition occurs at a finite interchain coupling. Relevances to the lightly doped quantum antiferromagnets and multi-layer quantum Hall systems are discussed.
\end{abstract}

PACS numbers: 75.10.Jm, 75.10.-b, 05.30.-d

Typeset using REVTEX 
Low-dimensional quantum antiferromagnets (AFM) exhibit many remarkable properties. In strictly one-dimension, transitions into ordered states with broken symmetry is absent. For the nearest-neighbor Heisenberg spin- $S$ chains, the low-lying excitations are gapless spin$1 / 2$ quanta (spinons) for half-odd-integer $S$, whereas a finite energy gap exists for integer $S$ [1]. This profound difference is captured in the effective $\mathrm{O}(3)$ nonlinear $\sigma$-model $(\mathrm{NL} \sigma \mathrm{M})$ description by the value of the topological angle $(\theta=2 \pi S)$ in the $1+1$-dimensional action [1].

In two-dimensions, spatially isotropic Heisenberg AFM on an unfrustrated lattice is proven rigorously to Néel order in the ground state for $S \geq 1$ [2]. While no such proof exists, it is widely believed that it is the case for $S=1 / 2$ as well. Indeed for $d \geq 2$, the long-wavelength, low-energy physics governing the interactions between the spin-waves in the ordered phase can be described by a $d+1$-dimensional NL $\sigma \mathrm{M}$ [3].

In this paper, we study quasi-one-dimensional quantum AFM, and in particular, the disorder-order transition associated with the dimensional crossover. Specifically, we consider $S=1 / 2$ antiferromagnetic (AF) spin chains with Heisenberg symmetry, arranged in a $d$-dimensional hypercubic lattice, and weakly-coupled by AF or ferromagnetic (FM) interchain exchange couplings $J_{\perp}$. Finite intrachain bond alternations are included to study the competition between magnetic order and dimerization. Our strategy is as follows. First, interchain coupling is considered at a mean-field level [6, [n in order to treat the important correlations that first develop along the strongly coupled chain-direction. The resulting effective one-dimensional theory is transformed into the massive Thirring model whose exact Bethe ansatz solution [6] is used to obtain the static and dynamical quantities. Then, we go beyond the mean-field theory, and show that the order parameter fluctuations can be described by an anisotropic $d+1$-dimensional NL $\sigma$ M. Renormalization group (RG) analyses are carried out to show that in the ground state, $d$-dimensional magnetic long-range order occurs for infinitesimal interchain coupling $\left|J_{\perp}\right|>0$. In the presence of bond alternation, long-range order develops when $J_{\perp}$ exceeds a finite critical value. Aside from obvious applications to real insulating compounds behaving as weakly coupled AF spin- $1 / 2$ chains at 
low-temperatures, we will discuss the implications of our results on the magnetic properties of underdoped insulating cuprates and point out the relevance to multi-layer quantum Hall structures.

The starting Hamiltonian of the system is given by

$$
H=J \sum_{i, r}\left[1+\delta(-1)^{i}\right] \mathbf{S}_{i, \mathbf{r}} \cdot \mathbf{S}_{i+1, \mathbf{r}}+J_{\perp} \sum_{i, \mathbf{r}, \mu} \mathbf{S}_{i, \mathbf{r}} \cdot \mathbf{S}_{i, \mathbf{r}+\mu},
$$

where $\mathbf{S}_{i, \mathbf{r}}$ is the spin-1/2 operator at lattice site $(i, \mathbf{r})$ with $i$ and $\mathbf{r}$ labeling the sites in the chain $(z)$ and transverse to the chain $\left(\mathbf{r}_{\mu}\right)$ directions, $\mu$ is summed over the $z_{\perp}=2(d-1)$ nearest-neighbors in the transverse directions. The intrachain exchange coupling is AF with alternating strengths $J(1 \pm \delta)>0$, whereas the interchain coupling can be either $\mathrm{AF}\left(J_{\perp}>0\right)$ or $\mathrm{FM}\left(J_{\perp}<0\right)$. We are interested in the case where $\delta,\left|J_{\perp}\right| / J \ll 1$.

The mean-field decoupling of the interchain term in Eq. (1) with respect to AF order in the $z$-direction in spin space leads to an effective Hamiltonian,

$$
H_{1 D}=J \sum_{i}\left[1+\delta(-1)^{i}\right] \mathbf{S}_{i} \cdot \mathbf{S}_{i+1}-h \sum_{i}(-1)^{i} S_{i}^{z},
$$

plus a constant term $H_{0}=z_{\perp} N_{s}\left|J_{\perp}\right| m_{0}^{2} / 2$. Here $N_{s}$ is the number of sites along the chain and $m_{0}=(-1)^{i}\left\langle S_{i}^{z}\right\rangle$ is the staggered magnetization. Eq. (22) describes a $1 \mathrm{D}$ AFM in a self-consistent staggered magnetic field $h=z_{\perp}\left|J_{\perp}\right| m_{0}$.

Next, we perform a standard Jordan-Wigner transformation $S_{i}^{z}=\psi_{i}^{\dagger} \psi_{i}-1 / 2, S_{i}^{+}=$ $\psi_{i}^{\dagger} \exp \left(i \pi \sum_{j=1}^{i-1} \psi_{j}^{\dagger} \psi_{j}\right)$. In terms of the usual left (L) and right (R) moving fermionic fields $\psi_{L}$ and $\psi_{R}$, the resulting theory in the continuum limit is given by

$$
\begin{aligned}
H_{1 D}^{\prime} & =\int d z\left[-i v\left(\psi_{L}^{\dagger} \partial_{z} \psi_{L}-\psi_{R}^{\dagger} \partial_{z} \psi_{R}\right)+2 g \psi_{L}^{\dagger} \psi_{R}^{\dagger} \psi_{R} \psi_{L}\right. \\
& \left.-h\left(\psi_{L}^{\dagger} \psi_{R}+\psi_{R}^{\dagger} \psi_{L}\right)+i \delta J\left(\psi_{L}^{\dagger} \psi_{R}-\psi_{R}^{\dagger} \psi_{L}\right)\right] .
\end{aligned}
$$

It is well known that the values of $v$ and $g$ obtained in the naive continuum limit are not correct in the Heisenberg limit. However, a comparison to the exact excitation spectrum of the Hamiltonian in Eq. (2) at $h=\delta=0$ [7] leads to $v=\pi J a / 2$, where $a$ is the lattice constant set to unity hereafter. The terms proportional to $h$ and $\delta J$ in Eq. (3) are easily seen 
in their bosonized forms to be relevant operators of dimension $x=1 / 2[8]$. These competing (AF order v.s. dimerization) interactions will induce a mass gap $(\Delta)$ with scaling exponent $1 /(2-x)$. Thus, $\Delta / v \propto(h / v, \delta)^{2 / 3}$.

Under a global chiral rotation: $\psi_{L} \rightarrow \exp (i \theta / 2) \psi_{L}, \psi_{R} \rightarrow \exp (-i \theta / 2) \psi_{R}$ with $\theta=$ $\tan ^{-1} \delta J / \Delta_{0}$ and $\Delta_{0}^{2}=h^{2}+\delta^{2} J^{2}$, terms proportional to $h$ and $\delta J$ transform into $-\Delta_{0}\left(\psi_{L}^{\dagger} \psi_{R}+\right.$ $\left.\psi_{R}^{\dagger} \psi_{L}\right)$. The resulting Hamiltonian is then identical to the massive Thirring model with bare mass $\Delta_{0}$ and interaction $g$, which was solved by Bethe ansatz [6]. Following Ref. [4], we obtain $g=2 v=\pi J$ and the ground state energy gain per site due to $m_{0}$,

$$
\Delta E=z_{\perp}\left|J_{\perp}\right| m_{0}^{2} / 2-J\left(7 / 10 \pi^{1 / 3}\right)\left(\Delta_{0} / J\right)^{4 / 3}
$$

The self-consistent value for the staggered magnetization is obtained by minimizing $\Delta E$ with respect to $m_{0}$. We find a critical value for the interchain coupling,

$$
\left|J_{\perp}^{c}\right|=\left(15 \pi^{1 / 3} / 14 z_{\perp}\right) J \delta^{2 / 3}
$$

which separates an AF phase for $\left|J_{\perp}\right|>\left|J_{\perp}^{c}\right|$ where the renormalized mass gap $\Delta=$ $(14 \sqrt{3} / 5 \pi) z_{\perp}\left|J_{\perp}\right|$ and

$$
m_{0}=\left(\frac{z_{\perp}}{\pi}\right)^{1 / 2}\left(\frac{14}{15}\right)^{3 / 2}\left|\frac{J_{\perp}}{J}\right|^{1 / 2}\left(1-\left|\frac{J_{\perp c}}{J_{\perp}}\right|^{3}\right)^{1 / 2},
$$

from a dimerized phase for $\left|J_{\perp}\right|<\left|J_{\perp}^{c}\right|$ where $\Delta_{\text {dis }}=\left(3 \sqrt{3} / \pi^{2 / 3}\right) J \delta^{2 / 3}$ and $m_{0}=0$.

The mean-field theory predicts a Néel temperature $T_{N} \propto z_{\perp} J_{\perp}$ in the ordered phase. While this can be correct when the coordination number $z_{\perp}$ is large, it obviously contradicts the Mermin-Wagner theorem, i.e. AF long-range order should not be possible at any finite temperature in $d=2$. It is thus necessary to go beyond the mean-field theory and include the order parameter fluctuations. To this end, we turn to the dynamical spin correlations in the ordered phase. Note that the ordering wavevector $\mathbf{Q}=\left(\mathbf{Q}_{\perp}, \pi\right)$ where $\mathbf{Q}_{\perp}=(\pi, \pi, \ldots)$ for $J_{\perp}>0$ and $\mathbf{Q}_{\perp}=(0,0, \ldots)$ for $J_{\perp}<0$. Since the translation symmetry is broken, the uniform and the staggered components of the spins are coupled by umklapp scattering with momentum transfer $\mathbf{q} \rightarrow \mathbf{q}+\mathbf{Q}$. The transverse susceptibility in the random phase approximation is therefore given by a $2 \times 2$ matrix relation, 


$$
\chi(\mathbf{q}, \omega)=\chi^{0}\left(q_{z}, \omega\right)\left[\mathbf{1}-\left|J_{\perp}\right| f\left(\mathbf{q}_{\perp}\right) \chi^{0}\left(q_{z}, \omega\right)\right]^{-1}
$$

where $\mathbf{q}=\left(\mathbf{q}_{\perp}, q_{z}\right)$, and $f\left(\mathbf{q}_{\perp}\right)=\sum_{\mu} \exp \left(i \mathbf{q}_{\perp} \cdot \mu\right)$. Using the equations of motion obtained for $H_{1 D}$, and the Lorentz-invariance of $H_{1 D}^{\prime}$ valid at low energies $\omega \ll J$, it is straightforward to show that the components of the $1 \mathrm{D}$ susceptibility $\chi_{u u}^{0}, \chi_{u s}^{0}$, and $\chi_{s u}^{0}$ are entirely determined by $\chi_{s s}^{0}\left(q_{z}, \omega\right)$ in the long wavelength limit [4]. The latter has the following form,

$$
\chi_{s s}^{0}\left(q_{z}, \omega\right)=\frac{w}{\Delta^{2}+v^{2} q_{z}^{2}-\omega^{2}}+\mathcal{M}\left(\omega^{2}-v^{2} q_{z}^{2}\right)
$$

Here the pole in the first term arises from the lowest energy triplet excitation which corresponds to an added fermion in the Thirring model. The function $\mathcal{M}(x)$ contains the contributions from the continuum involving particle-hole excitations in the Thirring model. The latter has a threshold singularity at and a vanishing spectral weight below $\omega=2 \Delta$. Thus $\chi(\mathbf{q}, \omega)$ in Eq. (17) is dominated by the collective excitations for $\omega<2 \Delta$. We will neglect the contributions from $\mathcal{M}(x)$ in Eq. (8), which is equivalent to the single mode approximation (SMA). The constant $w$ in Eq. (8) is then fixed at $w=\Delta^{2} / z_{\perp}\left|J_{\perp}\right|$ by the condition $\chi_{s s}^{0}(0,0)=1 / z_{\perp}\left|J_{\perp}\right|$.

Solving for $\chi(\mathbf{q}, \omega)$ in Eq. (17) using the SMA, we obtain the staggered transverse susceptibility

$$
\chi_{s}(\mathbf{q}, \omega)=\frac{\Delta^{2}}{z_{\perp}\left|J_{\perp}\right|} \frac{1-h^{2} / \Delta^{2}}{\omega_{\mathbf{q}}^{2}-\omega^{2}}
$$

where $\omega_{\mathbf{q}}$ is the gapless spin wave dispersion (Goldstone modes), $\omega_{\mathbf{q}}^{2}=\bar{\Delta}^{2}\left(1-f\left(\mathbf{q}_{\perp}\right) / z_{\perp}\right)+$ $\bar{v}^{2} q_{z}^{2}$ in terms of the weakly modified mass gap $\bar{\Delta}=\Delta \sqrt{1-h^{2} / \Delta^{2}}$ and velocity $\bar{v}=$ $v \sqrt{1-h^{2} / \Delta^{2}}$ due to the interchain correlations [9]. The uniform static susceptibility,

$$
\chi_{\perp}=\frac{h^{2} / \Delta^{2}}{z_{\perp}\left|J_{\perp}\right|} \frac{1}{1-h^{2} / \Delta^{2}} .
$$

In the limit $\left|J_{\perp}\right| \rightarrow 0$ and $\delta=0, \chi_{\perp} \simeq 1.07\left(1 / \pi^{2} J\right)$. The close agreement of the latter with the exact $1 \mathrm{D}$ result $\left(1 / \pi^{2} J\right)$ suggests that the SMA is a rather accurate description. When $\delta \neq 0, \chi_{\perp}$ and thus the spin stiffness vanishes linearly as $\left|J_{\perp}\right| \rightarrow\left|J_{\perp}^{c}\right|$. 
The interactions between the AF spin waves can be described by the $\mathrm{O}(3)$ quantum $\mathrm{NL} \sigma \mathrm{M}$ [3]. From the susceptibilities derived above, the $d+1$-dimensional Euclidean action is given by (setting $\hbar=1$ ),

$$
\mathcal{S}_{0}=\frac{1}{2 g} \sum_{\left\langle\mathbf{r}, \mathbf{r}^{\prime}\right\rangle} \int d z \int_{0}^{\beta \Lambda \bar{v}} d \tau\left[\left(\partial_{z} \mathbf{n}_{\mathbf{r}}\right)^{2}+\left(\partial_{\tau} \mathbf{n}_{\mathbf{r}}\right)^{2}+\frac{R}{\Lambda^{2}}\left|\mathbf{n}_{\mathbf{r}}-\mathbf{n}_{\mathbf{r}^{\prime}}\right|^{2}\right] .
$$

Here $\mathbf{n}_{\mathbf{r}}(z, \tau)$ is a three-component unit-modulus vector field. It represents the local orientation of the AF order parameter. The discrete sum runs over the neighboring lattice sites in the transverse directions. As usual, $\beta=1 / k_{B} T$, and $\Lambda$ is a spatial cutoff at which the coupling constant $g=\bar{v} / \rho_{s}^{0}$ and $\rho_{s}^{0}=\chi_{\perp} \bar{v}^{2}$. The anisotropy is contained in $R=\Delta^{2} / z_{\perp} v^{2} \simeq 0.97 z_{\perp}\left(\left|J_{\perp}\right| / J\right)^{2} \ll 1$. In terms of the unit vector field, the transverse spin susceptibility $\chi_{s}(\mathbf{q}, \omega)=m_{0}^{2}\left\langle n^{+}(\mathbf{q}, \omega) n^{-}(\mathbf{q}, \omega)\right\rangle$ and the staggered magnetization $m=\left.m_{0}\left\langle n^{z}\right\rangle\right|_{T=0}$.

The RG analysis of $\mathcal{S}_{0}$ is subtle. Let us consider the case when $\delta=0$, i.e. for vanishing bond alternation. Notice that we did not keep track of the topological term explicitly since at $\theta=\pi$ it does not renormalize under the RG. The effect of the latter is however crucial for the renormalization of the coupling constant $g$ in the $1+1$-dimensional sector in the limit $R \rightarrow 0$ [10]. In the presence of the topological term, $g$ flows to a finite fixed point value $g(\infty)$ and the correlation length is infinite, whereas $g \rightarrow \infty$ and the system develops a finite correlation length $\xi_{\sigma} / \Lambda \approx e^{2 \pi / g}$ in its absence. Thus, during the RG transformation of Eq. (11), if the anisotropy is large enough such that $\sqrt{R} / \Lambda \ll 1 / \xi_{\sigma}$, further renormalization using the $d+1$-dimensional $\mathrm{RG}$ cannot eliminate this finite correlation length, the correct treatment of the $S=1 / 2$ system must include the effect of the topological term. On the other hand, in the opposite limit where $1 \gg \sqrt{R} / \Lambda \gg 1 / \xi_{\sigma}$, the long-wavelength physics is essentially controlled by the $d+1$-dimensional $\mathrm{RG}$ and the topological term would not make a qualitative difference in the ordered phase. Below, we consider the two situations separately.

For $\sqrt{R} \ll e^{-2 \pi / g}$, we follow the analysis of dimensional crossover [10, 11]. Since $R$ is exponentially small, the RG in the 1+1-dimensional sector can be performed independently 
by integrating out high-momentum modes until the effective couplings become comparable in all directions at a larger cutoff $\Lambda_{\perp}$, i.e. when $R / \Lambda^{2} g \approx 1 / \Lambda_{\perp}^{2} g\left(\Lambda_{\perp}\right)$. The $d+1$-dimensional RG is switched on thereafter. For large $\Lambda_{\perp}$, the coupling $g\left(\Lambda_{\perp}\right)$ flows towards its limiting fixed point value which is of order one. Thus, the crossover length scale is $\Lambda_{\perp} \approx \Lambda / \sqrt{R}$. Since the scaling dimension of the $\mathbf{n}$-field is zero in the ordered phase, there is no need to rescale the latter in the interchain term in Eq. (11). Taking the continuum limit in the transverse directions by absorbing the cutoff $\Lambda_{\perp}^{-2}$ into defining the derivatives, Eq. (11) is reduced to a continuous, isotropic action at an isotropic cutoff $\Lambda_{\perp}$,

$$
\mathcal{S}_{1}=\frac{1}{2 g_{d+1}} \int d^{d-1} \mathbf{r} \int d z \int_{0}^{\beta \Lambda_{\perp} \bar{v}} d \tau\left[\left(\partial_{\mathbf{r}} \mathbf{n}\right)^{2}+\left(\partial_{z} \mathbf{n}\right)^{2}+\left(\partial_{\tau} \mathbf{n}\right)^{2}\right],
$$

where $g_{\mathrm{d}+1}=g\left(\Lambda_{\perp}\right) \Lambda_{\perp}^{d-1}$ is the bare coupling constant for the $d+1$-dimensional RG. For $R \rightarrow 0, g\left(\Lambda_{\perp}\right) \rightarrow g(\infty)$. Thus, the stability of the AF ordered state at infinitesimal $R$ is determined by whether $g_{d+1}$ is smaller than the critical coupling $g_{d+1}^{c}$ of the $d+1$-dimensional RG. The latter predicts a $T=0$ fixed point at $g_{d+1}^{c} / \Lambda_{\perp}^{d-1}=(d-1) 2^{d} \pi^{d / 2} \Gamma(d / 2)$ to one-loop order [3].

Since the exact solution of $H_{1 D}$ in Eq. (ृ) describes the fixed point physics in the $1+1$ dimensional sector, $g(\infty)=1 / \chi_{\perp} \bar{v}$ can be calculated using the results in Eqs. (99) and (10). (i) For $\delta=0, g(\infty)=b\left[1-(2 / b \pi) z_{\perp}\left|J_{\perp}\right| / J\right]^{1 / 2}$ with $b=810 / 14 \pi^{2}$, which approaches the value $g(\infty)=5.86$ from below as $\left|J_{\perp}\right| \rightarrow 0$. Thus, the condition for AF order, $\bar{g}_{d+1} \equiv$ $g_{d+1}\left(\Lambda_{\perp}\right) / g_{d+1}^{c}<1$, is satisfied and improved further with increasing $z_{\perp}\left|J_{\perp}\right|$. This one-loop result is consistent with the numerical series expansion analysis in $d=2$ for AF coupled chains [10]. We therefore conclude that in the absence of bond alternation, long-range order develops for infinitesimal AF or FM interchain couplings in $d \geq 2$. The physical origin of this behavior should be traced back to the gapless power-law correlations in the spin-1/2 Heisenberg chain. For $d=2$, a finite temperature fixed point does not exist in the $\mathrm{NL} \sigma \mathrm{M}$, the ordered phase is stable only at $T=0$. This corrects the naive mean-field prediction of a finite $T_{N}$. (ii) When $\delta$ is finite, $\left|J_{\perp}^{c}\right| \neq 0$ in Eq. (5). For $z_{\perp}\left|J_{\perp}\right| / J \ll 1$, we find $g(\infty) \simeq b\left(1-\left|J_{\perp}^{c} / J_{\perp}\right|^{3}\right)^{-1}$ which diverges as $\left|J_{\perp}\right|$ is reduced toward $\left|J_{\perp}^{c}\right|$, indicating 
a transition into a disordered phase with finite dimerization. The critical coupling is at a $\left|J_{\perp}^{*}\right|\left(>\left|J_{\perp}^{c}\right|\right.$ predicted in the mean-field theory) where $\bar{g}_{d+1}=1$. To one-loop order, $\left|J_{\perp}^{*}\right|=\left|J_{\perp}^{c}\right|\left(1-\bar{g}_{d+1}^{\delta=0}\right)^{-1 / 3}$. The renormalized spin stiffness vanishes on approaching the transition according to $\rho_{s}=\rho_{s}^{0}\left(1-\bar{g}_{d+1}\right)$. Since $\left\langle\left(n^{z}\right)^{2}\right\rangle=\left(1-\bar{g}_{d+1}\right)$, the renormalized staggered magnetization $m^{2}=m_{0}^{2}\left(1-\bar{g}_{d+1}\right)$.

In the above discussion, the coupling constant $g$ defined at cutoff $\Lambda$ in Eq. (11) was taken to be close to the (order one) fixed point value of $H_{1 D}$. From the point of view of the effective $\mathrm{NL} \sigma \mathrm{M}$, this does not have to be the case. If $g \ll 1$, the independent $1+1$-dimensional RG should be replaced by the $d+1$-dimensional $R G$ once the reduced anisotropy is in the range $1 \gg \sqrt{R^{\prime}} \gg e^{-2 \pi / g\left(\Lambda^{\prime}\right)}$ at a cutoff $\Lambda^{\prime}=\Lambda \sqrt{R^{\prime} g / R g\left(\Lambda^{\prime}\right)}$. Further analysis then belongs to the second parameter regime which is also relevant for a large-S AFM where $g \sim 2 / S$.

For $1 \gg \sqrt{R} \gg e^{-2 \pi / g}$, because $R$ is no longer exponentially small, the $1+1$-dimensional RG does not transform independently. Instead, one should treat the $d+1$-dimensional RG with anisotropic cutoffs and expect the residual anisotropy to persist down to the $d+1$ dimensional fixed point. Taking the continuum limit in the transverse directions at cutoff $\Lambda_{\perp}=\Lambda / \sqrt{R}$ in Eq. (11) leads to an action like the one in Eq. (12) with bare coupling $g_{d+1}=g \Lambda_{\perp}^{d-1}$. The important difference is that the momentum cutoffs are now anisotropic, $\left|\mathbf{k}_{\perp}\right|<k_{\perp}^{m}=\pi / \Lambda_{\perp},\left|k_{z}\right|<k_{z}^{m}=\pi / \Lambda$. We have carried out the momentum shell RG by integrating out modes in the high-momentum layers of the $d$-dimensional box in $k$-space with $k_{\perp}^{m}>\left|\mathbf{k}_{\perp}\right|>k_{\perp}^{m} e^{-l}$ and $k_{z}^{m}>\left|k_{z}\right|>k_{z}^{m} e^{-l}$, where $e^{l}$ is the length rescaling factor. At $T=0$, the one-loop RG equation is $d g_{d+1} / d l=(1-d) g_{d+1}+g_{d+1}^{2} / g_{d+1}^{c}(R)$. The critical coupling $g_{d+1}^{c}(R)$ depends on the anisotropy $R$. For $d=2$, we find,

$$
g_{2+1}^{c}(R)=2 \pi \Lambda_{\perp} \sqrt{R}[\ln (\sqrt{1+R}+\sqrt{R})+\sqrt{R} \ln (\sqrt{1+1 / R}+1 / \sqrt{R})]^{-1} .
$$

For $R \ll 1, g_{2+1}^{c}(R) \approx 2 \pi \Lambda_{\perp} / \ln (2 / \sqrt{R})$. The ratio of the bare coupling to the fixed point value is thus $\bar{g}_{2+1}(R) \approx(g / 2 \pi) \ln (2 / \sqrt{R}) \ll 1$ in this parameter regime. In fact, for all $d \geq 2$ one can show $g_{d+1}^{c}(R) \propto \pi \Lambda_{\perp}^{d-1} / \ln (2 / \sqrt{R})$ such that $\bar{g}_{d+1}(R) \ll 1$. Thus the conclusion is again that the weakly-coupled spin chains are in the ordered phase. When 
$\delta \neq 0$, the uniform susceptibility $\chi_{\perp}$ decreases with decreasing $\left|J_{\perp}\right|$. Thus $g$ increases until $\sqrt{R} \sim e^{-2 \pi / g}$. The ordered phase becomes unstable at a critical value $R_{c}$ defined by $\bar{g}_{d+1}\left(R_{c}\right)=1$ where a transition into the dimerized phase takes place. Close to the transition, $\rho_{s}=\rho_{s}^{0} \sqrt{R}\left(1-\bar{g}_{d+1}(R)\right)$ and $m^{2}=m_{0}^{2}\left(1-\bar{g}_{d+1}(R)\right)$.

The finite temperature properties close to the transition are described by the scaling behavior of the $d+1$-dimensional quantum $\mathrm{NL} \sigma \mathrm{M}$. The correlation length $(\xi)$ at lowtemperatures can be obtained by integrating the one-loop RG equations [3]. For $d=2$, in the quantum critical regime, $\xi=\left(\hbar \bar{v} / k_{B} T\right) 2 \pi \Lambda / g_{2+1}\left(R_{c}\right)$ at $\bar{g}_{2+1}\left(R_{c}\right)=1$, where $\bar{v}$ is the effective spin-wave velocity along the chain direction. For $R_{c} \ll 1, \xi \propto\left(\hbar \bar{v} / k_{B} T\right) \sqrt{R_{c}}\left|\ln \sqrt{R_{c}}\right|$. In the renormalized classical regime, $\xi \propto \sqrt{R}|\ln \sqrt{R}|\left(\hbar \bar{v} / k_{B} T\right) \exp \left(2 \pi \rho_{s} / k_{B} T\right)$. Thus the effects of anisotropy enter the prefactors of these universal functions.

Recently, it was proposed that the destruction of AF long-range order in the lightlydoped insulating cuprates may be explained by the dimensional crossover in an effective low-energy theory of a 2D Heisenberg AFM with increasing anisotropy [12]. The important question is whether the effective spin quantum number of the AFM corresponds to an integer or a half-odd-integer. Since the effective theory is motivated by the physics of the striped phases [13] [15] with a large correlation length compared the distance between the stripes, the effective spin presumably depends on the number of spin- $1 / 2$ chains between the neighboring stripes. The latter is, unfortunately, not known precisely in the lightly-doped regime. It was shown in Ref. [12], that for integer effective spins (appropriate for the case of an even number of spins between the stripes), AF long-range order disappears below a finite critical interchain coupling related to Eq. (13). In the case where the effective spin corresponds to a half-integer, our results show that AF long-range order is stable for arbitrarily small interchain couplings in agreement with previous theoretical work [10]. In order to destroy AF order in this case, small dimerization is necessary in addition to anisotropy. Note that the amount of dimerization (bond alternation) necessary to disorder the system and open up a spin gap is $\delta_{c} \propto\left(J_{\perp} / J\right)^{3 / 2}$ which can be very small, whereas $\delta_{c} \approx 0.3$ on a dimerized square lattice Heisenberg AFM without spatial anisotropy [16]. 
The properties of the $S=1 / 2 \mathrm{AF}$ spin chains can provide useful insights into the integer quantum Hall transitions. In a single layer quantum Hall structure (QHS), the latter is in the universality class of the dimerization (spin-Peierls) transition of an $\mathrm{SU}(2 \mathrm{n}) \mathrm{AF}$ quantum spin chain in the limit $n \rightarrow 0$ [17]. The double-layer (or spin Landau level mixing) case corresponds to two FM coupled spin chains [18]. These results suggest qualitatively similar phase structures in the $n=1$ and $n \rightarrow 0$ cases. The difference in the universal properties of the phase transitions can be summarized by the changes in the critical exponents with n. A large number of coupled multi-layer QHS corresponds naturally to an array of FM coupled spin chains, where the interchain coupling originates from tunneling between the layers. The quasi-1D spin chains may order for infinitesimal $\left|J_{\perp}\right|>0$, as we have shown for $n=1$. The resulting spin wave spectrum just describes the diffusive modes that would appear in a disordered metal, suggesting the formation of a metallic phase between the insulator/quantum Hall states, consistent with recent numerical simulations [19]. The corresponding phase transitions are thus in the universality class of the $U(2 n) / U(n) \times\left. U(n)\right|_{n \rightarrow 0}$ $\mathrm{NL} \sigma \mathrm{M}$. Interestingly, the latter also describes the 3D Anderson transition in the presence of time-reversal symmetry breaking.

The author thanks A. H. Castro Neto for a discussion. This work was supported in part by an award from Research Corporation. 


\section{REFERENCES}

[1] F. D. M. Haldane, Phys. Lett. A93, 464 (1983); Phys. Rev. Lett.50, 1153 (1983). I. Affleck, Nucl. Phys. B257, 397 (1985); I. Affleck and F. D. M. Haldane, Phys. Rev. B36, 5291 (1987).

[2] T. Kennedy, E. H. Lieb, and B. S. Shastry, J. Stat. Phys. 53, 108 (1988).

[3] S. Chakravarty, B. I. Halperin, and D. R. Nelson, Phys. Rev. B39, 2344 (1989); Phys. Rev. Lett. 60, 1057 (1988).

[4] H. J. Schulz, Phys. Rev. Lett. 77, 2790 (1996).

[5] D. J. Scalapino, Y. Imry, and P. Pincus, Phys. Rev. B11, 2042 (1975).

[6] H. Bergknoff and H. B. Thacker, Phys. Rev. D19, 3666 (1979).

[7] R. J. Baxter, Ann. Phys. (N.Y.) 70, 193 (1972).

[8] I. Affleck, in Fields, Strings and Critical Phenomena, proceedings of the Les Houches summer school XLIX, eds. E. Brezin and J. Zinn-Justin (Elsevier, 1989).

[9] In deriving these results, we have dropped negligible contributions of $\mathcal{O}\left(\delta^{2}\left|J_{\perp}\right| / J\right)$ to the spin wave dispersion.

[10] I. Affleck and B. I. Halperin, preprint, cond-mat/9603078 (1996); I. Affleck, M. P. Gelfand and R. R. P. Singh, J. Phys. A27, 7313 (1994); Erratum 28, 1787 (1995).

[11] T. S. Chang and H. E. Stanley, Phys. Rev. B8, 4435 (1973); M. K. Grower, Phys. Lett. A44, 253 (1973).

[12] A. H. Castro Neto and D. Hone, Phys. Rev. Lett. 76, 2165 (1996).

[13] J. Zaanen and J. Gunnarson, Phys. Rev. B40, 7391 (1989); H. J. Schulz, J. Physique 50, 2833 (1989). S. A. Kivelson and V. J. Emery, in Proceedings of Strongly Correlated Electronic Materials: The Los Alamos Symposium 1993. edited by K. S. Bedell et al. ( 
Addison-Wesley, 1994), and references therein.

[14] J. M. Tranquada et al, Nature (London) 375, 561 (1995).

[15] F. Borsa, et. al. Phys. Rev. B52, 7334 (1995).

[16] N. Katoh and M. Imada, J. Phys. Soc. Jpn. 62, 3728 (1993).

[17] D-H Lee, Phys. Rev. B50, 10788 (1994); D-H Lee and Z. Wang, Phil. Mag. Lett. 73, $145(1996)$.

[18] Z. Wang, D.-H. Lee, and X.-G. Wen, Phys. Rev. Lett. 72, 2454 (1994).

[19] J. T. Chalker and A. Dohmen, Phys. Rev. Lett. 75, 4496 (1995). 